Mark McClure, Department of Mathematics, Washington and Lee

University, Lexington, VA 24450, email: mmcclure@@wlu.edu

\title{
ENTROPY DIMENSIONS OF THE HYPERSPACE OF COMPACT SETS
}

\begin{abstract}
Let $(X, \rho)$ be a metric space, let $(\mathcal{K}(X), \widetilde{\rho})$ denote the space of nonempty compact subsets of $X$ with the Hausdorff metric, and let $E \subset X$. The purpose of this paper is to investigate the relationships between the entropy dimensions of $E$ and of $\mathcal{K}(E)$.
\end{abstract}

\section{The Definition and Properties of the Entropy Indices.}

Given a separable metric space $(X, \rho)$, let $\mathcal{K}(X)$ denote the set of non-empty compact subsets of $X$. Define a metric $\widetilde{\rho}$ on $\mathcal{K}(X)$ as follows: For $A, B \in \mathcal{K}(X)$ let

$$
\widetilde{\rho}(A, B)=\max \left\{\sup _{x \in A}\{\operatorname{dist}(x, B)\}, \sup _{y \in B}\{\operatorname{dist}(y, A)\}\right\} .
$$

The space $(\mathcal{K}(X), \widetilde{\rho})$ is called the Hausdorff metric space, or hyperspace, associated with $X$ and inherits several nice geometrical properties from $X$. For example, $\mathcal{K}(X)$ is complete whenever $X$ is complete and $\mathcal{K}(X)$ is compact whenever $X$ is compact. A discussion of the Hausdorff metric including proofs of the above is in [Ed] section 2. 4. To avoid confusion between metric spaces and their corresponding hyperspaces, tildes will be used to denote reference to the hyperspace. So for example, if $A \subset X$ is compact and $\varepsilon>0$, then $\widetilde{B}_{\varepsilon}(A) \subset \mathcal{K}(X)$ denotes the closed ball of radius $\varepsilon$ about the set $A$.

The upper and lower entropy dimensions, denoted by $\widehat{\Delta}$ and $\widehat{\delta}$ respectively, are defined in terms of the upper and lower entropy indices denoted by $\Delta$ and $\delta$. In this section the upper and lower entropy indices will be defined in a

Key Words: fractal dimensions, entropy dimension, hyperspace

Mathematical Reviews subject classification: Primary: 28C20, 28D20, 54B20

Received by the editors November 21, 1994 
way suitable for investigating infinite dimensional spaces and some of their basic properties will be developed. The earliest references on the entropy indices are $[\mathrm{Bou}]$ and $[\mathrm{PonSc}]$. The indices were generalized and studied on various spaces of functions in [KolTi]. The modification to obtain the entropy dimensions goes back at least to [Weg]. The treatment here has also been influenced by [Ed] and [Tr]. Initially, it must be assumed that $E$ is totally bounded to define $\Delta$ and $\delta$. For every $\varepsilon>0$, let

$$
N_{\varepsilon}(E)=\max \# \text { of disjoint closed balls centered in } E \text { with radius } \varepsilon / 2 \text {. }
$$

Such a collection of balls will be called an $\varepsilon$-packing of $E$. The idea behind the entropy indices is that the asymptotic behavior of $N_{\varepsilon}(E)$ as $\varepsilon \searrow 0$ should be indicative of the dimension of $E$. For example, if $I^{n}$ is an $n$-dimensional cube, then $N_{\varepsilon}\left(I^{n}\right) \asymp(1 / \varepsilon)^{n}$ ([KolTi] sec. 4). The notion of a Hausdorff function provides a convenient measure of the asymptotic behavior of $N_{\varepsilon}$. A Hausdorff function is a function $\varphi:[0, \infty) \rightarrow[0, \infty)$ which is continuous, non-decreasing, and satisfies $\varphi(0)=0$. Let $\Phi$ denote the set of all Hausdorff functions. The upper entropy index $\Delta$ is defined to be a partition of $\Phi$ obtained by comparing the asymptotic behavior of $N_{\varepsilon}(E)$ as $\varepsilon \rightarrow 0$ to that of Hausdorff functions. Specifically, for totally bounded $E$ write

$$
\Delta(E)=\left(\Phi_{\infty}(E), \Phi_{+}(E), \Phi_{0}(E)\right),
$$

where

$$
\begin{aligned}
\Phi_{\infty}(E) & =\left\{\varphi \in \Phi: \limsup _{\varepsilon \searrow 0} \varphi(\varepsilon) N_{\varepsilon}(E)=\infty\right\}, \\
\Phi_{+}(E) & =\left\{\varphi \in \Phi: 0<\limsup _{\varepsilon \searrow 0} \varphi(\varepsilon) N_{\varepsilon}(E)<\infty\right\}, \\
\Phi_{0}(E) & =\left\{\varphi \in \Phi: \limsup _{\varepsilon \searrow 0} \varphi(\varepsilon) N_{\varepsilon}(E)=0\right\} .
\end{aligned}
$$

The lower entropy index, $\delta$, is defined in a similar way, but with the lim sup replaced by the liminf. Ideally one would like to completely describe this partition, but it is more tractable to concentrate on one particular Hausdorff function or family of Hausdorff functions. The following abbreviations will, hopefully, make the statement of the theorems presented here more natural. For $\varphi \in \Phi$ write

- $\Delta(E) \succ \varphi$ if $\varphi \in \Phi_{\infty}(E)$

- $\Delta(E) \asymp \varphi$ if $\varphi \in \Phi_{+}(E)$

- $\Delta(E) \prec \varphi$ if $\varphi \in \Phi_{0}(E)$ 
- $\Delta(E) \succeq \varphi$ if $\varphi \in \Phi_{\infty}(E) \cup \Phi_{+}(E)$

- $\Delta(E) \preceq \varphi$ if $\varphi \in \Phi_{0}(E) \cup \Phi_{+}(E)$.

The above symbols will also be used to compare Hausdorff functions. So write:

- $\varphi \prec \psi$ if $\lim _{t \searrow 0} \frac{\psi(t)}{\varphi(t)}=0$,

- $\varphi \preceq \psi$ if $\lim \sup _{t \searrow 0} \frac{\psi(t)}{\varphi(t)}<\infty$,

- $\varphi \asymp \psi$ if $0<\liminf _{t \searrow 0} \frac{\psi(t)}{\varphi(t)} \leq \lim \sup _{t \searrow 0} \frac{\psi(t)}{\varphi(t)}<\infty$.

Note that if $\varphi \prec \psi \preceq \Delta(E)$, then $\varphi \prec \Delta(E)$ since

$$
N_{\varepsilon}(E) \varphi(\varepsilon)=N_{\varepsilon}(E) \psi(\varepsilon) \frac{\varphi(\varepsilon)}{\psi(\varepsilon)} \rightarrow 0
$$

as $\varepsilon \rightarrow 0$. Thus, if the entropy indices are thought of as notions of dimension, then $\prec$ places a partial order on $\Phi$ in which the faster a function disappears at the origin, the larger dimension.

The relationship between $\Delta(E)$ and $\Delta(\mathcal{K}(E))$ is described by the following lemmas. Note that all that follows holds for $\delta$ with very similar proofs.

Lemma 1.1 For totally bounded $E \subset X, \Delta(E) \succ \varphi$ implies $\Delta(\mathcal{K}(E)) \succ$ $2^{-1 / \varphi}$.

Proof. For $\varepsilon>0$, let $\mathcal{B}_{\varepsilon}=\left\{B_{\varepsilon / 2}\left(x_{i}\right)\right\}_{i=1}^{N_{\varepsilon}(E)}$ be an $\varepsilon$-packing of $E$ with $N_{\varepsilon}(E)$ balls of radius $\varepsilon / 2$. If $F \subset\left\{x_{i}\right\}_{i=1}^{N_{\varepsilon}(E)}$ is non-empty, then $F \in \mathcal{K}(E)$ and we may consider $\widetilde{B}_{\varepsilon / 2}(F) \subset \mathcal{K}(E)$, the ball of radius $\varepsilon / 2$ centered on $F$. Note that

$$
\widetilde{B}_{\varepsilon / 2}(F)=\left\{C \in \mathcal{K}(E): C \subset \cup_{x_{i} \in F} B_{\varepsilon / 2}\left(x_{i}\right) \text { and } C \cap B_{\varepsilon / 2}\left(x_{i}\right) \neq \emptyset \forall x_{i} \in F\right\} .
$$

From this it is easy to see that given distinct, non-empty $F_{1}, F_{2} \subset\left\{x_{i}\right\}_{i=1}^{N_{\varepsilon / 2}(E)}$ we have $\widetilde{B}_{\varepsilon / 2}\left(F_{1}\right) \cap \widetilde{B}_{\varepsilon / 2}\left(F_{2}\right)=\emptyset$. So $N_{\varepsilon}(\mathcal{K}(E)) \geq 2^{N_{\varepsilon}(E)}-1$ and

$$
\begin{aligned}
N_{\varepsilon}(\mathcal{K}(E)) 2^{-\frac{1}{\varphi(\varepsilon)}} & \geq\left(2^{N_{\varepsilon}(E)}-1\right) 2^{-\frac{1}{\varphi(\varepsilon)}}=2^{N_{\varepsilon}(E)-\frac{1}{\varphi(\varepsilon)}}-2^{\frac{-1}{\varphi(\varepsilon)}} \\
& =2^{\frac{N_{\varepsilon}(E) \varphi(\varepsilon)-1}{\varphi(\varepsilon)}}-2^{\frac{-1}{\varphi(\varepsilon)}} .
\end{aligned}
$$

Thus

$$
\limsup _{\varepsilon \rightarrow 0} N_{\varepsilon}(\mathcal{K}(E)) 2^{-\frac{1}{\varphi(\varepsilon)}} \geq \limsup _{\varepsilon \rightarrow 0}\left(2^{\frac{N_{\varepsilon}(E) \varphi(\varepsilon)-1}{\varphi(\varepsilon)}}-2^{\frac{-1}{\varphi(\varepsilon)}}\right)=\infty,
$$


since $\lim \sup _{\varepsilon \rightarrow 0} N_{\varepsilon}(E) \varphi(\varepsilon)=\infty$. So $\Delta(\mathcal{K}(E)) \succ 2^{-1 / \varphi}$.

For the reverse inequality it will be useful to define the quantity $M_{\varepsilon}$ by

$$
M_{\varepsilon}(E)=\text { min number of sets of diameter } \leq \varepsilon \text { needed to cover } E \text {. }
$$

$M_{\varepsilon}$ is related to $N_{\varepsilon}$ as in the following lemma.

Lemma 1.2 Let $E$ be totally bounded and let $\varepsilon>0$. Then

$$
N_{\varepsilon}(E) \leq M_{\varepsilon / 2}(E) \leq N_{\varepsilon / 4}(E) .
$$

Proof. The first inequality holds because any two closed disjoint balls of radius $\varepsilon / 2$ must have centers separated by more than $\varepsilon / 2$. Thus an $\varepsilon / 2$-cover requires at least one set for each element of an $\varepsilon$-packing.

The second inequality follows from the fact that a maximal $\varepsilon / 4$-packing induces an $\varepsilon / 2$-cover by doubling the radius of each of the balls.

Lemma 1.3 Let $E$ be totally bounded. If $\Delta(E) \prec \varphi(\varepsilon)$, then $\Delta(\mathcal{K}(E)) \prec$ $2^{-1 / \varphi(\varepsilon / 4)}$.

Proof. Let $\mathcal{B}_{\varepsilon}=\left\{B_{\varepsilon / 2}\left(x_{i}\right)\right\}_{i=1}^{N_{\varepsilon}(E)}$ be an $\varepsilon$-packing of $E$. Then

$$
\widetilde{\mathcal{B}}_{\varepsilon}=\left\{\widetilde{B}_{\varepsilon}(F)\right\}_{F \subset\left\{x_{i}\right\}_{i}, F \neq \emptyset}
$$

forms a $2 \varepsilon$-cover of $\mathcal{K}(E)$ with $2^{N_{\varepsilon}(E)}-1$ elements. So

$$
N_{4 \varepsilon}(\mathcal{K}(E)) \leq M_{2 \varepsilon}(\mathcal{K}(E)) \leq 2^{N_{\varepsilon}(E)}-1
$$

for every $\varepsilon>0$. So

$$
\begin{aligned}
N_{4 \varepsilon}(\mathcal{K}(E)) 2^{-1 / \varphi(\varepsilon)} & \leq\left(2^{N_{\varepsilon}(E)}-1\right) 2^{-1 / \varphi(\varepsilon)} \\
& =2^{\frac{N_{\varepsilon}(E) \varphi(\varepsilon)-1}{\varphi(\varepsilon)}}-2^{-1 / \varphi(\varepsilon)} \rightarrow 0
\end{aligned}
$$

as $\varepsilon \rightarrow 0$. Thus $\Delta(\mathcal{K}(E)) \prec 2^{-1 / \varphi(t / 4)}$.

The following corollary is somewhat more concrete.

Corollary 1.1 If $E$ is totally bounded and $\Delta(E) \asymp \varepsilon^{s_{0}}$ (finite dimension $s_{0}$ ), then

$$
\Delta(\mathcal{K}(E)) \begin{cases}\prec 2^{-(1 / \varepsilon)^{s}} & \text { for } s>s_{0} \\ \succ 2^{-(1 / \varepsilon)^{s}} & \text { for } s<s_{0} .\end{cases}
$$


Proof. If $s<s_{0}$, then $\Delta(E) \succ \varepsilon^{s}$. So $\Delta(\mathcal{K}(E)) \succ 2^{-(1 / \varepsilon)^{s}}$, by Lemma 1.1.

If $s_{0}<s_{1}<s$, then $\Delta(E) \prec \varepsilon^{s_{1}}$. So $\Delta(\mathcal{K}(E)) \prec 2^{-(4 / \varepsilon)^{s_{1}}} \prec 2^{-(1 / \varepsilon)^{s}}$, by Lemma 1.3.

Equation 3.29 and theorem 9.3 of [Fal] show that this corollary applies to a self-similar set $E \subset \mathbb{R}^{n}$ of finite upper entropy index $s_{0}$. Analogous statements for the Hausdorff dimension of the hyperspace of such sets will be studied in a subsequent paper $([\mathrm{Mcc}])$.

It is natural to ask if $\Delta(E) \asymp \varphi$ implies $\Delta(\mathcal{K}(E)) \asymp 2^{-1 / \varphi}$. The answer is no, basically because the $\asymp$ relation is too sensitive. For example, if $I \subset \mathbb{R}$ is a closed interval of length $\ell$, then the optimal $\varepsilon$-packing of $I$ consists of $[\ell / \varepsilon]$ balls with centers separated by $\varepsilon$. So $N_{\varepsilon}(I)=[\ell / \varepsilon]$ and $\lim _{\varepsilon \rightarrow 0} \varepsilon N_{\varepsilon}(I)=\ell$. Thus $\Delta(I) \asymp \varepsilon$ for any bounded interval $I$. But, as we see in the proofs of Lemmas 1.1 and 1.3,

$$
2^{N_{\varepsilon}(E)}-1 \leq N_{\varepsilon}(\mathcal{K}(E)) \leq 2^{N_{\varepsilon / 4}(E)}-1
$$

So

And

$$
N_{\varepsilon}(\mathcal{K}(I)) 2^{-1 / \varepsilon} \begin{cases}\geq\left(2^{[\ell / \varepsilon]}-1\right) 2^{-1 / \varepsilon} \rightarrow \infty & \text { if } \ell>1 \\ \leq\left(2^{[4 \ell / \varepsilon]}-1\right) 2^{-1 / \varepsilon} \rightarrow 0 & \text { if } \ell<\frac{1}{4}\end{cases}
$$

$$
\Delta(\mathcal{K}(I)) \begin{cases}\succ 2^{-1 / \varepsilon} & \text { if } \ell>1 \\ \prec 2^{-1 / \varepsilon} & \text { if } \ell<\frac{1}{4} .\end{cases}
$$

\section{The Definition and Properties of the Entropy Dimen- sions.}

A disadvantage with the entropy indices is that they are not $\sigma$-stable. It is possible that $\Delta\left(\cup_{1}^{\infty} E_{n}\right) \succ \varphi$ even if $\Delta\left(E_{n}\right) \prec \varphi$ for every $n$ as the following example shows.

Example 2.1 Let $\varphi \in \Phi$ and $a_{n} \in \varphi^{-1}(\{1 / n\})$. Note that $a_{n} \searrow 0$. Let $X=\left\{x_{1}, x_{2}, \ldots, x_{\infty}\right\}$ be a countable set. Define a metric $\rho$ on $X$ by

$$
\rho\left(x_{n}, x_{m}\right)=\left\{\begin{array}{cl}
a_{n} & \text { if } n \neq m=\infty \\
a_{n}+a_{m} & \text { if } \infty \neq n \neq m \neq \infty \\
0 & \text { if } n=m .
\end{array}\right.
$$

Of course, $X$ is a countable union of singletons each satisfying $\Delta\left(\left\{x_{n}\right\}\right) \prec \psi$ for any $\psi \in \Phi$. On the other hand, the $n$ balls of radius $\varepsilon / 2$ centered at $x_{1}, \ldots, x_{n}$ form an $\varepsilon / 2$ packing of $X$ whenever $a_{n+1}<\varepsilon \leq a_{n}$. Hence,

$$
N_{\varepsilon}(X) \varphi(\varepsilon) \geq n \varphi(\varepsilon) \geq \frac{n}{n+1} \rightarrow 1
$$


as $n \rightarrow \infty$ and $\delta(X) \succeq \varphi$.

The $\sigma$-stable upper entropy dimension is defined to be the partition of $\Phi$,

$$
\widehat{\Delta}(E)=\left(\widehat{\Phi}_{\infty}(E), \widehat{\Phi}_{+}(E), \widehat{\Phi}_{0}(E)\right)
$$

obtained from $\Delta(E)$ in the following way:

$$
\begin{gathered}
\varphi \in \widehat{\Phi}_{\infty} \text { if } \forall \text { decomposition } E=\cup_{n=1}^{\infty} E_{n} \exists n \ni \Delta\left(E_{n}\right) \succ \varphi, \\
\varphi \in \widehat{\Phi}_{0} \text { if } \exists \text { a decomposition } E=\cup_{n=1}^{\infty} E_{n} \ni \Delta\left(E_{n}\right) \prec \varphi \forall n, \\
\widehat{\Phi}_{+}(E)=\Phi \backslash\left(\widehat{\Phi}_{\infty}(E) \cup \widehat{\Phi}_{0}(E)\right) .
\end{gathered}
$$

A similar procedure defines $\widehat{\delta}$ in terms of $\delta$. As with the entropy indices, the following abbreviations make the statements of theorems more natural. Write

- $\widehat{\Delta}(E) \succ \varphi$ if $\varphi \in \widehat{\Phi}_{\infty}(E)$,

- $\widehat{\Delta}(E) \asymp \varphi$ if $\varphi \in \widehat{\Phi}_{+}(E)$,

- $\widehat{\Delta}(E) \prec \varphi$ if $\varphi \in \widehat{\Phi}_{0}(E)$.

Some comments on these definitions are in order.

1. Let $F \subset E$. Since a packing by $\varepsilon / 2$-balls of $F$ is also one of $E$, the set functions $\Delta, \widehat{\Delta}, \delta$, and $\widehat{\delta}$ are all monotone. That is if $F \subset E$, then $\Delta(E) \prec \varphi$ implies $\Delta(F) \prec \varphi$.

2. Although $\Delta$ and $\delta$ are defined only for totally bounded sets, $\widehat{\Delta}$ and $\widehat{\delta}$ are defined for $\sigma$-totally bounded sets.

3. An $\varepsilon$-packing of $\bar{E}$ may be approximated by an $\varepsilon$-packing of $E$, which implies $N_{\varepsilon}(E)=N_{\varepsilon}(\bar{E})$. So $\Delta$ and $\delta$ respect closure and the sets $E_{n}$ in the definition above may be assumed to be closed.

The following lemma establishes $\sigma$-stability for $\widehat{\Delta}$. An analogous statement and proof is valid for $\widehat{\delta}$.

Lemma 2.1 If $E=\cup_{n} E_{n}$ and $\widehat{\Delta}(E) \succ \varphi$, then there exists an $n$ such that $\widehat{\Delta}\left(E_{n}\right) \succ \varphi$. 
Proof. Suppose that each $E_{n}$ is further decomposed $E_{n}=\cup_{k} E_{n, k}$. Then $E=\cup_{n} \cup_{k} E_{n, k}$. Since $\widehat{\Delta}(E) \succ \varphi$, there exists $n$ and $k$ such that $\Delta\left(E_{n, k}\right) \succ \varphi$. $E_{n, k}$ is part of an arbitrary decomposition of $E_{n}$. This says that this $n$ satisfies $\widehat{\Delta}\left(E_{n}\right) \succ \varphi$.

The main goal of this paper is to investigate the relationship between $\widehat{\Delta}(E)$ and $\widehat{\Delta}(\mathcal{K}(E))$. The following theorem addresses one direction of this relationship. Again, the corresponding statement and proof hold for $\widehat{\delta}$.

Theorem 2.1 If $E$ is a $\sigma$-compact subset of a metric space $X$ and $\widehat{\Delta}(E) \succ \varphi$, then $\widehat{\Delta}(\mathcal{K}(E)) \succ 2^{-1 / \varphi}$.

Proof. Suppose that $\widehat{\Delta}(E) \succ \varphi$ and $E$ is compact. The extension of the theorem to $\sigma$-compact sets is straightforward as any such set contains a compact subset $E$ satisfying $\widehat{\Delta}(E) \succ \varphi$ by $\sigma$-stability. A subset of $E$ with a certain regularity property will need to be highlighted. Let

$$
E_{\varphi}=\left\{x \in E: \widehat{\Delta}\left(E \cap B_{r}(x)\right) \succ \varphi \forall r>0\right\} .
$$

$E \backslash E_{\varphi}$ is open in $E$ practically by definition. $E_{\varphi}$ is seen to be non-empty as follows. Assume to the contrary and choose for every $x \in E$ an $r_{x}>0$ such that $\widehat{\Delta}\left(B_{r_{x}}(x) \cap E\right) \nsucc \varphi$. Then $\left\{B_{r_{x}}(x)\right\}_{x \in E}$ forms an open cover of the compact set $E$. So there is a finite subcover say $\left\{B_{r_{i}}\left(x_{i}\right)\right\}_{i=1}^{n}$. Then, for each $i=1, \ldots, n$ we have $\widehat{\Delta}\left(B_{r_{i}}\left(x_{i}\right) \cap E\right) \nsucc \varphi$ contrary to $\sigma$-stability. So let $x \in E_{\varphi}$ and let $r>0$. The next step is to show that $\widehat{\Delta}\left(E_{\varphi} \cap B_{r}(x)\right) \succ \varphi$. (This is the needed regularity property of $E_{\varphi}$.) First, $\widehat{\Delta}\left(E \cap B_{r}(x)\right) \succ \varphi$ by definition. For every $y \in\left(E \backslash E_{\varphi}\right) \cap B_{r}(x)$, choose an $r_{y}$ such that $\widehat{\Delta}\left(E \cap B_{r_{y}}(y)\right) \nsucc \varphi$. Then $\left\{B_{r_{y}}(y)\right\}_{y \in\left(E \backslash E_{\varphi}\right) \cap B_{r}(x)}$ is an open cover of the $\sigma$-compact set $\left(E \backslash E_{\varphi}\right) \cap B_{r}(x)$. So there is a countable subcover $\left\{B_{r_{y_{k}}}\left(y_{k}\right)\right\}_{k=1}^{\infty}$. Since

$$
E \cap B_{r}(x)=\left(\cup_{k=1}^{\infty} B_{r_{y_{k}}}\left(y_{k}\right) \cap E\right) \cup\left(E_{\varphi} \cap B_{r}(x)\right)
$$

and $\widehat{\Delta}\left(B_{r_{y_{k}}}\left(y_{k}\right) \cap E\right) \nsucc \varphi$ for any $k$, we have that $\widehat{\Delta}\left(E_{\varphi} \cap B_{r}(x)\right) \succ \varphi$ by $\sigma$-stability of $\widehat{\Delta}$.

It will now be shown that $\widehat{\Delta}\left(\mathcal{K}\left(E_{\varphi}\right)\right) \succ 2^{-1 / \varphi}$ from which it easily follows that $\widehat{\Delta}(\mathcal{K}(E)) \succ 2^{-1 / \varphi}$. Suppose that $\mathcal{K}\left(E_{\varphi}\right)=\cup_{1}^{\infty} \widetilde{K}_{n}$. It may be assumed that each $\widetilde{K}_{n}$ is closed. By the Baire category theorem, applicable since $\mathcal{K}(X)$ is complete, one of the $\widetilde{K}_{n}$ 's is somewhere dense. This means that there is a set $D \in \widetilde{K}_{n}$ and an $r>0$ such that $\widetilde{B}_{r}(D) \subset \widetilde{K}_{n}$. Let $x \in D$ and define

$$
\widetilde{A}_{x, r}=\left\{C \in \widetilde{K}_{n}: C=\left(D \backslash B_{r}\right) \cup F \text {, where } F \subset B_{r / 2}(x) \cap E_{\varphi} \text { is compact }\right\} .
$$


$\widetilde{A}_{x, r} \subset \widetilde{K}_{n}$ is naturally isometric to $\mathcal{K}\left(E_{\varphi} \cap B_{r / 2}(x)\right)$ which satisfies

$$
\Delta\left(\mathcal{K}\left(E_{\varphi} \cap B_{r / 2}(x)\right)\right) \succ 2^{-1 / \varphi},
$$

since $\Delta\left(E_{\varphi} \cap B_{r / 2}(x)\right) \succ \varphi$. Therefore, $\Delta\left(\widetilde{K}_{n}\right) \succ 2^{-1 / \varphi}$ and $\widehat{\Delta}\left(\mathcal{K}\left(E_{\varphi}\right)\right) \succ$ $2^{-1 / \varphi}$.

The next example shows that the converse inequality does not hold.

Example 2.2 Let $\varphi \in \Phi$ and let $X$ be as in Example 2.1. Then $\widehat{\Delta}(X) \prec \psi$ for every $\psi \in \Phi$ as $X$ is the countable union of singletons. But $\widehat{\Delta}(\mathcal{K}(X)) \succ 2^{-1 / \tau}$ for any $\tau \prec \varphi$.

Proof. Write $\mathcal{K}(X)=\widetilde{K} \cup \widetilde{I}$ where

$$
\widetilde{K}=\left\{C \in \mathcal{K}(X): x_{\infty} \in C\right\} \text { and } \widetilde{I}=\left\{C \in \mathcal{K}(X): x_{\infty} \notin C\right\} .
$$

$\widetilde{I}$ consists of the countably many isolated points of $\mathcal{K}(X)$. So, $\widetilde{K}$ is closed in $\mathcal{K}(X)$. Any decomposition of $\mathcal{K}(X)$ induces one of $\widetilde{K}$. So suppose that $\widetilde{K}=\cup_{n=1}^{\infty} \widetilde{K}_{n}$, where each $\widetilde{K}_{n}$ may be assumed closed. By the Baire Category Theorem, one of the $\widetilde{K}_{n}$ is somewhere dense. Thus there exists $n \in \mathbb{N}, C \in \widetilde{K}_{n}$, and $r>0$ such that $\widetilde{B}_{r}(C) \subset \widetilde{K}_{n}$. Let

$$
X_{r}=\left\{x \in X: \rho\left(x, x_{\infty}\right) \leq r\right\}, \quad C_{r}=\left\{x \in C: \rho\left(x, x_{\infty}\right)>r\right\},
$$

and let

$$
\widetilde{A}=\left\{D \in \mathcal{K}(E): D=C_{r} \cup F \text { where } F \in \mathcal{K}\left(X_{r}\right)\right\}
$$

Then $\widetilde{A} \subset \widetilde{B}_{r}(C) \subset \widetilde{K}_{n}$ and $\widetilde{A}$ is naturally isometric to $\mathcal{K}\left(X_{r}\right)$. Now $\Delta\left(X_{r}\right) \succeq$ $\varphi \succ \tau$ by Example 2.1. Hence $\Delta\left(\mathcal{K}\left(X_{r}\right)\right) \succ 2^{-1 / \tau}$ by Lemma 1.1. Thus $\widehat{\Delta}(\mathcal{K}(X)) \succ 2^{-1 / \tau}$.

In spite of this example we do have the following lemma, which says that if the set $E$ is "regular enough", then the expected type of upper bound holds.

Lemma 2.2 Suppose that $E$ is totally bounded and $\Delta(E) \prec \varphi(\varepsilon)$. Then $\widehat{\Delta}(\mathcal{K}(E)) \prec 2^{-1 / \varphi(\varepsilon / 4)}$.

Proof. This follows immediately from Lemma 1.3.

As with the entropy indices, these results are perhaps more clearly illustrated by considering finite dimensional sets.

Corollary 2.1 If $E$ is totally bounded and satisfies $\widehat{\Delta}(E) \asymp \varepsilon^{s_{0}} \asymp \Delta(E)$, then

$$
\widehat{\Delta}(\mathcal{K}(E)) \begin{cases}\prec 2^{-(1 / \varepsilon)^{s}} & \text { for } s>s_{0} \\ \succ 2^{-(1 / \varepsilon)^{s}} & \text { for } s<s_{0} .\end{cases}
$$


Proof. If $s_{0}<s_{1}<s$, then $\Delta(E) \prec \varepsilon^{s_{1}}$. So $\Delta(\mathcal{K}(E)) \prec 2^{-(4 / \varepsilon)^{s_{1}}} \prec 2^{-(1 / \varepsilon)^{s}}$, by Lemma 2.2 .

If $s<s_{0}$, then $\widehat{\Delta}(E) \succ \varepsilon^{s}$ and $\widehat{\Delta}(\mathcal{K}(E)) \succ 2^{-(1 / \varepsilon)^{s}}$, by Theorem 2.1.

Equation 3.29 and theorem 9.3 of [Fal] show that this corollary applies to a self-similar set $E \subset \mathbb{R}^{n}$ of finite upper entropy dimension $s_{0}$.

\section{References}

[Bou] G. Bouligand, Ensembles impropres et nombre dimensionnel. Bulletin des Sciences Mathématiques. 52 (1928) 320-344, 361-376.

[Ed] G. A. Edgar, Measure, Topology, and Fractal Geometry. Springer-Verlag, 1990.

[Fal] Kenneth Falconer, Fractal Geometry: Mathematical Foundations and Applications. John Wiley \& Sons, 1990.

[KolTi] A. N. Kolmogorov and V. M. Tihomirov, $\varepsilon$-entropy and $\varepsilon$-capacity of sets in functional spaces. AMS Trans. Series 2. 17 (1961), 277-364.

[Mcc] Mark McClure, The Hausdorff Dimension of the Hyperspace of Compact Sets., Real Analysis Exchange, Submitted.

[PonSc] L. Pontrjagin and L. Schnirelmann, Sur une propriété métrique de la dimension. Annals of Math. 33 (1932) 156-162.

[Tr] C. Tricot, Two definitions of fractional dimension. Math. Proc. Camb. Phil. Soc. 91 (1982), 57-74.

[Weg] H. Wegmann, Die Hausdorff-Dimension von kartesischen Produkten metrischer Räume. Journal fur die Reine und Angewandte Mathematik, 235 (1971) 46-75. 